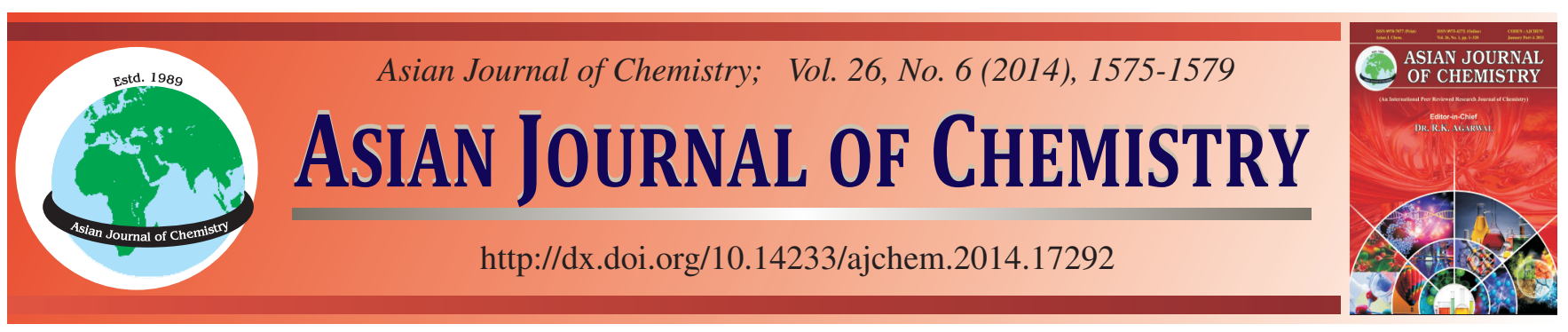

\title{
Visible Light Driven Catalytic Properties Over Methyl Orange by Novel $\mathrm{PtSe}_{2} /$ Graphene Nanocomposites $\dagger$
}

\author{
Kefayat Ullah, Sun Bok Jo, Shu Ye, Lei Zhu and Won-Chun OH*
}

Department of Advanced Materials Science \& Engineering, Hanseo University, Seosan-si, Chungnam-do, 356-706, Republic of Korea

*Corresponding author: Fax: +82 41 6883352; Tel: +82 41 6601337; E-mail: we_oh@hanseo.ac.kr

High efficient graphene based platinum selenide composite photocatalyst were synthesized by ultrasonic assisted method. The "asprepared" nanocomposites are further characterized by X-ray diffraction, scanning electron microscopy with an energy dispersive X-ray analysis, transmission electron microscopy, UV-visible absorbance spectra analysis and diffuse reflectance spectra analysis. The photocatalytic activities of the composites are investigated by the degradation of methyl orange as a standard dye. The results showed that the photocatalytic activity of $\mathrm{PtSe}_{2}$ was significantly enhanced by the loading of graphene in the composites.

Keywords: Graphene, Photocatalysis, Ultrasonics, Nanocomposites, Methyl orange.

\section{INTRODUCTION}

Graphene is the most emergent material on the perspective of nanotechnology and materials science ${ }^{1}$. Recently this 2D material is used in many technological applications such as sensors, supercapacitors, solar cell, hydrogen production and photocatalysis. In catalysis technology graphene based metallic and semiconductor materials are widely used in the degradation of environmental pollutants. Photocatalysis involves the in situ generation of oxidizing agents such as hydroxyl radicals. These radicals has the natural properties to degrade organic pollutants in water to obtain a complete mineralization into $\mathrm{CO}_{2}$, water and mineral acids such as sulfuric, hydrochloric and nitric $\operatorname{acids}^{2-4}$. In this context Pt/graphene nanocomposites were synthesized via microwave techniques and enhanced catalytic activity were observed towards the degradation of organic pollutants ${ }^{5}$. CdS nanorods graphene nanosheet were synthesized using one step method and enhanced catalytic activity were observed ${ }^{6}$. Several approaches have been reported that graphene can be used as good support for semiconductor nanocrystals due to unique electronic, mechanical, physical and chemical properties ${ }^{7-9}$. Flat monolayer and due to enormous charge transferring abilities and two dimensional structure of graphene make it suitable material to attach $\mathrm{PtSe}_{2}$ nanoparticles in order to improve the photocatalytic properties.
Many approaches, such as hydrothermal methods ${ }^{10}$, sol-gel methods, microwave assisted method ${ }^{11,12}$, have been developed to decorate graphene sheets with nanoparticles. Ultrasonication is an effective technique for attachment of nanoparticles on grephene sheet. Ultrasonication involves the use of ultrasound as a source of high energy at a frequency range of $18-100 \mathrm{kHz}$ causing the creation of a phenomenon called acoustic cavitations. It involves the formation, growth and collapse of cavity bubbles that entraps dissolved gases or vapors surrounding water ${ }^{13,14}$. Ultrasonication has been found important in using for initiation or enhancement of catalytic reaction in both homogeneous and heterogeneous cases ${ }^{15}$.

In this manuscript $\mathrm{PtSe}_{2}$ /graphene nanocomposites are prepared via a relatively facile ultrasoniction method. Attachment of $\mathrm{PtSe}_{2}$ on graphene nanosheet in aqueous solution was achieved in single step during the ultrasonic treatment.

\section{EXPERIMENTAL}

Graphene oxide was prepared in the laboratory following the Hummer's-Offeman method ${ }^{16}$. Hydrogen hexachloroplatinate(IV) hydrate $\left(\mathrm{H}_{2} \mathrm{PtCl}_{6} \cdot \mathrm{nH}_{2} \mathrm{O} ; \mathrm{n}=5.5\right)$ was used as a platinum source purchased from Kojima Chemical Co. Ltd., Japan. Selenium powder (Se), methyl orange (MO, $\left.\mathrm{C}_{14} \mathrm{H}_{14} \mathrm{~N}_{3} \mathrm{O}_{3} \mathrm{~S}\right)$ were used as model pollutant purchased from Samchun Pure Chemical Co. Ltd., Korea. In addition sodium sulphite

Fresented at The 7th International Conference on Multi-functional Materials and Applications, held on 22-24 November 2013, Anhui University of Science \& Technology, Huainan, Anhui Province, P.R. China 
$\left(\mathrm{Na}_{2} \mathrm{SO}_{3} \cdot 7 \mathrm{H}_{2} \mathrm{O}\right)$ was purchased from Dae-Jung Chemical and Metals Co. Ltd. Korea. All the chemicals were used as recevied and all experiments were carried out using distilled water.

Synthesis of $\mathrm{PtSe}_{2}$ : For the synthesis of $\mathrm{PtSe}_{2}$ precursor $2 \mathrm{~g}$ of anhydrous sodium sulphite $\left(\mathrm{Na}_{2} \mathrm{SO}_{3}\right)$ and $0.2 \mathrm{~g}$ of crude selenium powder were vigourously stirred in $100 \mathrm{~mL}$ of distilled water for $0.5 \mathrm{~h}$ to ensure the homogenous mixing. Followed by addition of hydrogen hexachloroplatinate $\left(\mathrm{H}_{2} \mathrm{PtCl}_{6} \cdot \mathrm{nH}_{2} \mathrm{O} ; \mathrm{n}=5.5\right)$ suspension in $20 \mathrm{~mL}$ water. The resulting reaction mixture was then ultrasonicated for $90 \mathrm{~min}$ at $35^{\circ} \mathrm{C}$ and allowed to cool at room tempertaure. The $\mathrm{PtSe}_{2}$ obtained precipitate was filtered using Whatman filter paper. The residue was washed several time with distilled water and dried in vacume oven at $90^{\circ} \mathrm{C}$ for $8 \mathrm{~h}$.

Synthesis of $\mathrm{PtSe}_{2}$ /graphene nanocomposites: $\mathrm{PtSe}_{2} /$ graphene nanocomposites were prepared with ultrasonication method. In this process, $1 \mathrm{~g}$ of graphene oxide was mixed with previously obtained $\mathrm{PtSe}_{2}(0.1 \mathrm{~mol})$ in $200 \mathrm{~mL}$ distilled water. The suspension was ultrasonicated for $3 \mathrm{~h}$ at $35^{\circ} \mathrm{C}$ and cooled at room temperature. The resultant solution was filtered and washed 3 times with distilled water and transferred into a dry oven for $6 \mathrm{~h}$ at $90^{\circ} \mathrm{C}$. The powder was then treated at 500 ${ }^{\circ} \mathrm{C}$ for $1 \mathrm{~h}$ in electric furnace to obtain $0.1 \mathrm{~mol} \mathrm{PtSe} /$ graphene nano composites. Same procedure was followed to obtain 0.2 mol of $\mathrm{PtSe}_{2}$ /graphene nanocomposits. The obtained samples were labeled as PSG1 and PSG2.

Dye absorption experiments using visible light: Photocatalytic activity of $\mathrm{PtSe}_{2}$ /graphene synthesized via ultrasonication method was tested by using methyl orange as an organic dye. The initial and final concentrations of methyl orange solution were determined by measuring absorbance changes at their respective absorption maxima at $464 \mathrm{~nm}$. In each test, $10 \mathrm{mg}$ of catalytic sample were taken in $80 \mathrm{~mL}$ solution of organic dyes $\left(0.1 \mathrm{mg} \mathrm{mL}^{-1}\right)$. The solution was stirred on a magnetic stirrer at room temperature for several minutes moderately and kept in a dark for $2 \mathrm{~h}$ to maintain the adsorption/desorption equilibrium of the dye. After $2 \mathrm{~h}$, the solution was exposed to visible light source which was kept at $20 \mathrm{~cm}$ above the solution. Samples were collected after 30, 60, 90, 120, 150 and 180 min and centrifuged for $10 \mathrm{~min}$ instantly to remove the solid material from the solution for further analysis. The photocatalytic behaviours of the samples were analyzed by the absorbance spectrometry with UV/visible spectrophotometer (Optizen POP, Mecasys, Korea).

The crystal structures and phases of the samples were obtained by XRD (Shimata XD-D1, Japan) with $\mathrm{CuK}_{\alpha}$ radiation $(\lambda=1.54056 \AA)$ in the range of $2 \theta$ from $10-80^{\circ}$ at a scan speed of $1.2^{\circ} \mathrm{m}^{-1}$. The morphology of the samples was studied by SEM (JSM-5200 JOEL, Japan). Energy dispersive $\mathrm{X}$-ray spectroscopy (EDX) was also employed for elemental analysis. Transmission electron microscopy (TEM, JEOL, JEM-2010, Japan) was used to observe the surface state and structure of the photocatalyst composites at an acceleration voltage of $200 \mathrm{kV}$. TEM was also used to examine the size and distribution of the $\mathrm{PtSe}_{2}$ particles on graphene sheet. Diffuse reflectance spectra (DRS) were obtained by using a scan UV/visible spectrophotometer (Neosys-2000) equipped with an integrating sphere assembly. The decomposition kinetics for the photocatalytic activity was measured by using spectrometer (Optizen POP, Mecasys, Korea).

\section{RESULTS AND DISCUSSION}

Growth and characterization: The XRD pattern of the $\mathrm{PtSe}_{2}$ /graphene nanocomposites synthesized via ultrasonic assisted method is shown in Fig. 1. The (002) of graphene peaks are located at $26^{\circ}$ and $25^{\circ}$ at $2 \theta$ the intensity of the peaks are very small as compared to $\mathrm{PtSe}_{2}$ peaks. This suppression of the graphene peak confirms that $\mathrm{PtSe}_{2}$ nanocrystals are well developed on the graphene sheet. The crystalline $\mathrm{PtSe}_{2}$ exhibit the characteristic (001), (011), (012), (110), (111), (201), (202), (113), (121) and (122) reflections that correspond to hexagonal crystal phase having (JCPDS PDF\#: 00-065-3374). The average crystal size of $\mathrm{PtSe}_{2}$ and $\mathrm{PtSe}_{2} /$ graphene nanocomposites were calculated using scherrer equation based on the XRD peak broadening of (101) peak. The average particle sizes for the composite using Scherrer equation were in the range of 16-25 nm, which is much smaller than the bare $\mathrm{PtSe}_{2}$ $(36 \mathrm{~nm})$. The morphology of the $\mathrm{PtSe}_{2} /$ graphene nanocomposite synthesized via ultrasonication is shown in Fig. 2. From the SEM image shown in Fig. 2(a), the flaky structure of graphene is clearly observed reflecting its layered structure. During ultrasonication the graphene sheet is further broke off into thin small sheet pattern grown in different direction. The sheets are broken normally at the end point of the grain boundaries where the cracks occur and spread out in all directions ${ }^{17}$. In Fig. 2(b), it is worth noting that graphene sheet anchoring $\mathrm{PtSe}_{2}$ nanoparticle using ultrasonication as compare to bare graphene in Fig. 2(a). The sheet morphology is retained after ultrasonic treatment. It can be seen from Fig. 2(c) that $\mathrm{PtSe}_{2}$ nanoparticles occupy most of the available surface area of the graphene sheets, giving much higher loading to the $\mathrm{PtSe}_{2}$ nanoparticles in the composites. The nanoscale images of $\mathrm{PtSe}_{2}$ decorated graphene were taken by TEM. Fig. 3(a) clearly demonstrates that $\mathrm{PtSe}_{2}$ nanoparticles are dispersed throughout the surface of transparent graphene sheets indicating a strong interaction between graphene sheet and $\mathrm{PtSe}_{2}$ particles. In Fig. 3(b) well dispersed nanoparticles are clearly revealed on the graphene sheet having average particle size $25 \mathrm{~nm}$. It is well known that for removal of environmental pollutants an efficient photocatalyst should have a high photocatalytic activity in both UV and visible region, so that the whole solar-energy spectrum can be utilized to a large extent as possible. Diffuse reflectance spectra spectra of the PSG2 photocatalyst were recorded and shown in Fig. 4. From this figure it is clearly seen that the composite exhibit a large absorption in the visible range of the spectrum. The diffuse reflectance spectra of the PSG2 was transformed by performing the Kubelka-Munk transformation of the measured reflectance according to the following equation, the absorption coefficient is related to the band gap $\left(\mathrm{E}_{\mathrm{g}}\right)$ by the relation:

$$
\alpha=\frac{\alpha_{0}}{E}\left(E-E_{g}\right)^{\eta}
$$

where $\alpha_{o}$ is a constant and $\mathrm{E}$ is the incident photon energy. The constant $\eta$ depends on the type of transitions involved and its value is $\eta=1 / 2$ for direct allowed transitions. In order 


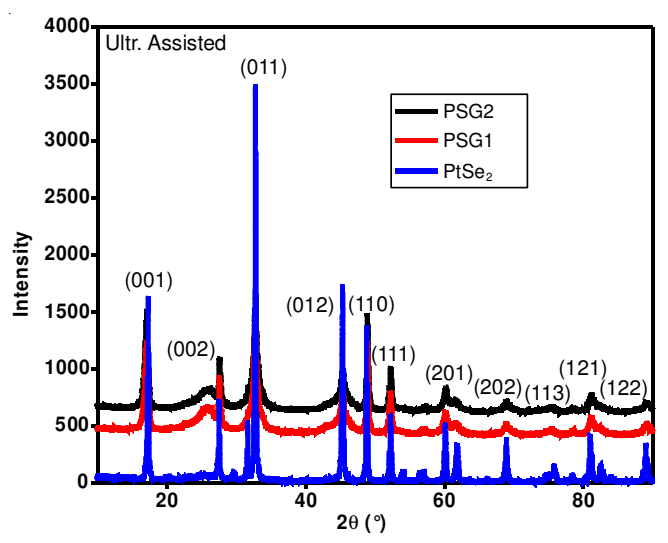

Fig. 1. XRD Pattern of $\mathrm{PtSe}_{2} /$ graphene nanpcomposites
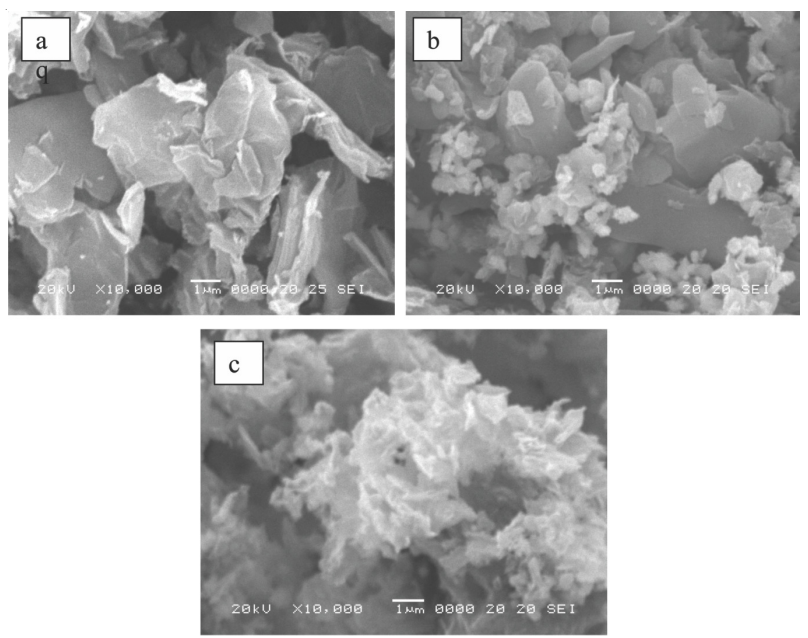

Fig. 2. SEM micrograps (a) graphene (b) PSG1 and (c) PSG2
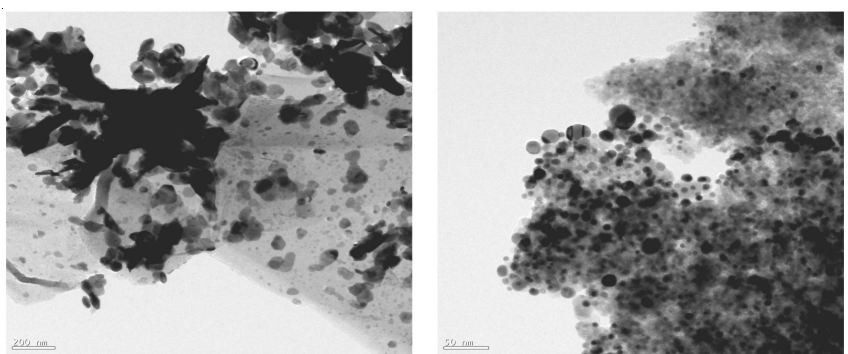

Fig. 3. TEM Images of PSG2 nanocomposites; (a) large scale and (b) small scale

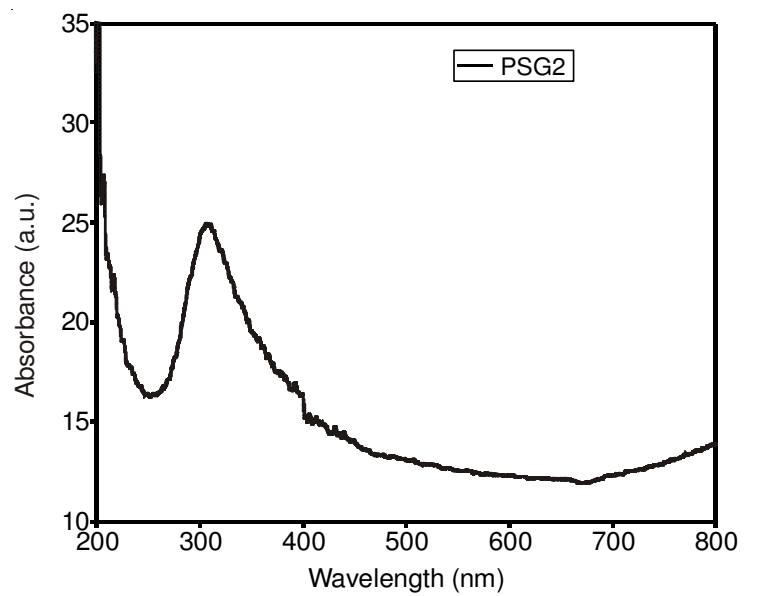

Fig. 4. DRS absorbance spectra of PSG2 nanocomposites to obtain the band gap, $(\alpha \mathrm{E})^{2}$ is plotted as a function of photon energy. The linear portions of the curves are fitted using linear regression analysis. An extrapolation of the linear regions of the plots gives the value of the band gap as the intercept to the horizontal axis (where $\alpha \mathrm{E}=0$ ). Such plots are shown in Fig. 5. The band gap of PSG1 and PSG2 nanocomposites was found to be and $1.89 \mathrm{eV}$. We found that by decorating graphene with $\mathrm{PtSe}_{2}$ nanoparticles gives a large absorption edge which lies in the visible range. The broad absorption is likely to arise from aggregation effects as the particles become densely crowded on the graphene sheet ${ }^{18}$. Thus, it can be inferred that decorating graphene with a metal selenide such as $\mathrm{PtSe}_{2}$ is effective for visible light response and can play a significant role in enhancing the photocatalytic activity ${ }^{19}$.

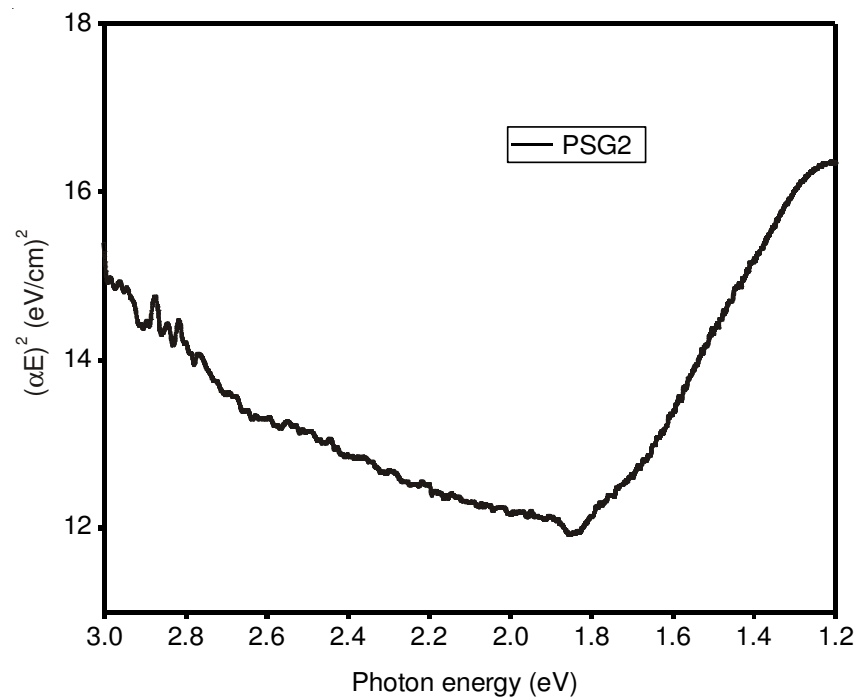

Fig. 5. Plots of $(\alpha \mathrm{E})^{2}$ versus photon energy for band gap determination

Photocatalytic properties: The photocatalytic performance of the $\mathrm{PtSe}_{2} /$ graphene nanocomposites in terms of photo degradation of methyl orange molecules under visible light irradiation is investigated. Three steps are mainly required for photocatalytic mechanism. The absorption of the pollutants, light absorption by the catalytic material used and charge transport. Carbon materials have astonishing absorption properties therefore mainly used in various environmental applications. Most of the industrial dyes are aromatic in nature and they create $\pi-\pi$ stacking interaction with the graphene aromatic domains. The concentration of the organic molecule increases at the surface of the photocatalytic materials due to absorption process. By the irradiation of light electron are excited from the valance band of $\mathrm{PtSe}_{2}$ to conduction band creating hole in the valence band. The photo excited electron and holes react with water molecule to create a radical oxygen species which further decompose the pollutants. The relation between the concentration of the adsorbent and the capture efficiency was investigated. As shown in Fig. 6, the characteristic UV/visible absorption intensity of methyl orange at $464 \mathrm{~nm}$ decreased on increasing the time interval. This shows the degradation efficiencies of the PSG2 nanocomposites. The normalized temporal concentration change of methyl orange during the photodegradation process is shown in Fig. 7. From Fig. 7, maximum degradation is observed for the PSG2 


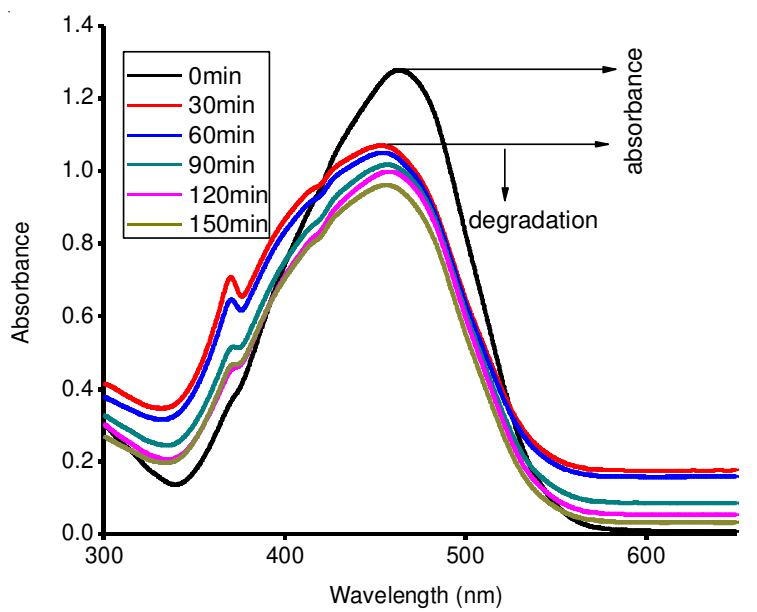

Fig. 6. UV/visible absorption spectra for the methyl orange degradation by PSG2 under visible light

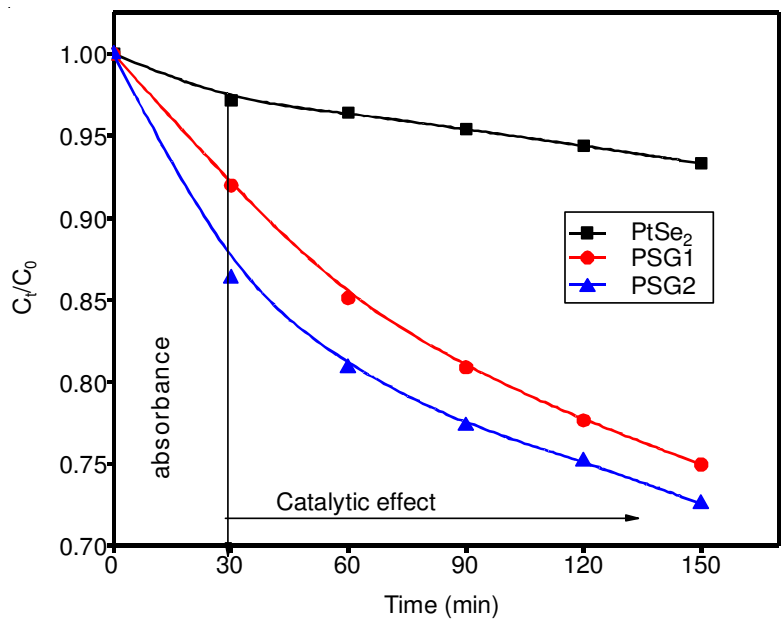

Fig. 7. Plot of methyl orange degradation efficiency $v s$. irradiation time

nanocomposites which are attributed to the uniform dispersion of $\mathrm{PtSe}_{2}$ nanoparticles on graphene sheet providing large number of reaction sites. The reactions between the dye molecules and the catalyst materials could be expressed by LangmuirHinshelwood model ${ }^{16}$. Fig. 8 implies that the degradation of methyl orange follows the pseudo first-order kinetics expressed as:

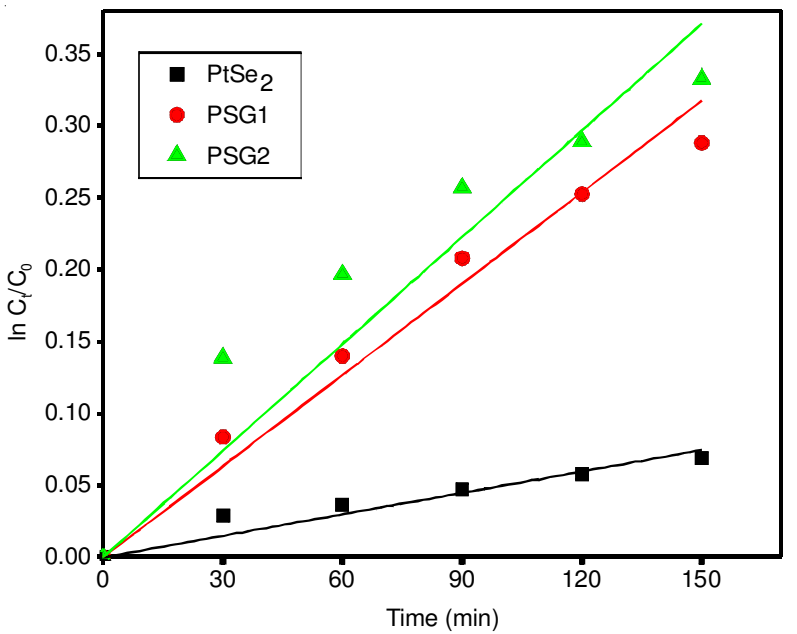

Fig. 8. Apparent pseudo- first order kinetics of methyl orange degradation over $\mathrm{PtSe}_{2} /$ graphene nanocomposites

$$
-\frac{\mathrm{dC}}{\mathrm{dt}}=\mathrm{K}_{\mathrm{app}} \mathrm{C}
$$

Integration this equation (with the restriction $\mathrm{C}=\mathrm{C}_{0}$ at $\mathrm{t}=$ 0 , with $\mathrm{C}_{0}$ being the initial concentration in the bulk solution after dark adsorption and $t$ is the reaction time) will lead to the following relation

$$
-\ln \frac{\mathrm{C}_{\mathrm{t}}}{\mathrm{C}_{0}}=\mathrm{K}_{\mathrm{app}} \mathrm{t}
$$

where $\mathrm{C}_{\mathrm{t}}$ and $\mathrm{C}_{0}$ are the reactant concentrations at times $\mathrm{t}=\mathrm{t}$, respectively and $\mathrm{K}_{\text {app }}$ and $\mathrm{t}$ are the apparent reaction rate constant and time, respectively. So from the graphical plot in Fig. 8 the slope of the linear plots should be equal to apparent first order rate constant $\left(\mathrm{K}_{\mathrm{app}}\right)$. The $\left(\mathrm{K}_{\mathrm{app}}\right)$ values give us the degradation rate of methyl orange by the photocatalyst materials under the influence of visible light. The rate constant $\left.\mathrm{K}_{(\text {app }}\right)$ for nanocomposites (PtSe 2 , PSG1, PSG2) are $\left(4.9 \times 10^{-4}\right.$, $\left.2.1 \times 10^{-3}, 2.47 \times 10^{-3}\right)$, respectively. The above results suggest that the PSG2 composite is better photocatalyst among the three samples. The scheme for charge transfer between $\mathrm{PtSe}_{2}$ nanoparticles and graphene is shown in Fig. 9. Due to the small band gap energy of $\mathrm{PtSe}_{2}$, incident light irradiation produces electrons $\left(\mathrm{e}^{-}\right)$in the conduction band $(\mathrm{CB})$ and holes $\left(\mathrm{h}^{+}\right)$in the valence band (VB). Thus, a number of electrons $\left(\mathrm{e}^{-}\right)$and holes $\left(\mathrm{h}^{+}\right)$were generated in $\mathrm{PtSe}_{2}$. Meanwhile, graphene nanosheets transfer electrons $\left(\mathrm{e}^{-}\right)$to the conduction band of $\mathrm{PtSe}_{2}$, thereby increasing the number of electrons as well as the rate of electron-induced redox reactions. The graphene coupled $\mathrm{PtSe}_{2}$ system shows enhanced catalytic activity due to high charge seperation induced by the synergitic effects of graphene and $\mathrm{PtSe}_{2}$. The generated electrons $\left(\mathrm{e}^{-}\right)$react with dissolved oxygen molecules and produce oxygen peroxide radicals $\mathrm{O}_{2}{ }^{-}$. The positive charge hole $\left(\mathrm{h}^{+}\right)$can react with $\mathrm{OH}^{-}$ derived from $\mathrm{H}_{2} \mathrm{O}$ to form hydroxyl radicals $\mathrm{OH}^{*}$. The organic dyes used may degraded by oxygen peroxide radicals $\mathrm{O}_{2}{ }^{--}$and hydroxyl radicals $\mathrm{OH}^{\bullet}$ to $\mathrm{CO}_{2}, \mathrm{H}_{2} \mathrm{O}$ and other mineralization products ${ }^{20,21}$. The reactions involved in the charge mobility and mineralization of the dyes are as follows:

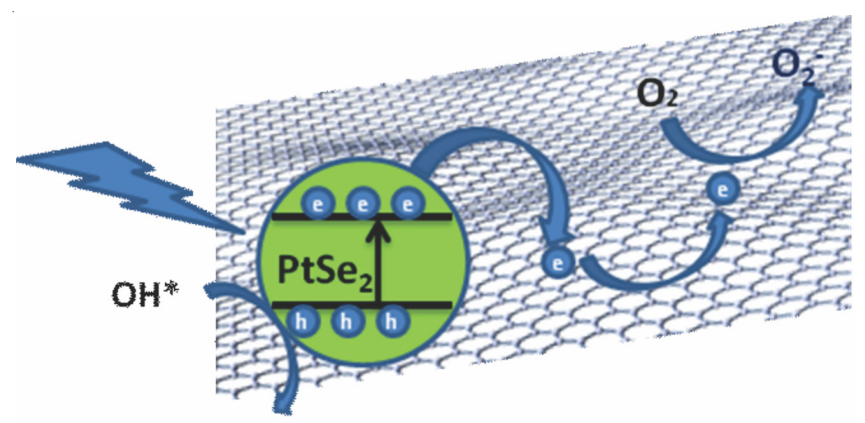

$\mathrm{OH}-$

Fig. 9. Schematic diagram for the generation of electrons and holes on the interface of $\mathrm{PtSe}_{2} /$ graphene nanocomposites

(hv) $\mathrm{PtSe}_{2} /$ graphene $\rightarrow \mathrm{PtSe}_{2} /$ graphene $\left(\mathrm{h}^{+}+\mathrm{e}^{-}\right)$

$$
\begin{gathered}
\mathrm{h}^{+}+\mathrm{OH}^{-} \rightarrow \mathrm{OH}^{\bullet} \\
\mathrm{O}_{2}+\mathrm{e}^{-} \rightarrow \mathrm{O}_{2}^{--} \\
(\mathrm{MO})+\mathrm{OH}^{\bullet}+\mathrm{O}_{2}^{--} \rightarrow \mathrm{CO}_{2}+\mathrm{H}_{2} \mathrm{O} \\
+ \text { other mineralized by products }
\end{gathered}
$$




\section{Conclusion}

In this work, we have synthesized $\mathrm{PtSe}_{2}$ /graphene nanocomposite by a facile ultrasonic assisted technique. It is clearly observed from the TEM images that most of the $\mathrm{PtSe}_{2}$ nanoparticles are well dispersed on graphene sheet without agglomeration. The DRS results give a large absorption edge which is evident that our nanocomposite can be used as visible light driven photocatalyst materials. The catalytic activities of $\mathrm{PtSe}_{2}$ /graphene composites were examined by degradation of methyl orange in aqueous solutions under visible light irradiation. The degradation experiments proved the influence of graphene support in dye removal. Enhanced photcatalytic activities were observed towards decolorization of methyl orange by $\mathrm{PtSe}_{2} /$ graphene nanocomposites.

\section{REFERENCES}

1. Y. Zhang and C. Pan, Diamond Rel. Mater., 24, 1 (2012).

2. C. Lee, X. Wei, J.W. Kysar and J. Hone, Science, 321, 385 (2008).

3. M.D. Stoller, S. Park, Y. Zhu, J. An and R.S. Ruoff, Nano Lett., 8, 3498 (2008).

4. A.A. Balandin, S. Ghosh, W. Bao, I. Calizo, D. Teweldebrhan, F. Miao and C.N. Lau, Nano Lett., 8, 902 (2008).

5. K.I. Bolotin, K.J. Sikes, Z. Jiang, M. Klima, G. Fudenberg, J. Hone, P. Kim and H.L. Stormer, Solid State Commun., 146, 351 (2008).
6. X. Du, I. Skachko, A. Barker and E.Y. Andrei, Nat. Nanotechnol., 3, 491 (2008).

7. C. Stampfer, E. Schurtenberger, F. Molitor, J. Guttinger, T. Ihn and K. Ensslin, Nano Lett., 8, 2378 (2008).

8. K. Haubner, J. Murawski, P. Olk, L.M. Eng, C. Ziegler, B. Adolphi and E. Jaehne, ChemPhysChem, 11, 2131 (2010).

9. F. Lupo, R. Kamalakaran, C. Scheu, N. Grobert and M. Rühle, Carbon, 42, 1995 (2004).

10. X.Y. Zhang, H.P. Li, X.L. Cui and Y.H. Lin, J. Mater. Chem., 20, 2801 (2010).

11. A.Z. Abdullah and P.Y. Ling, J. Hazard. Mater., 173, 159 (2010).

12. K. Ullah, L. Zhu, Z.-D. Meng, S. Ye, Q. Sun and W.-C. Oh, Chem. Eng. J., 231, 76 (2013).

13. N.H. Ince and G. Tezcanli-Güyer, Ultrasonics, 42, 591 (2004).

14. G.A. Tai and W.L. Guo, Ultrason. Sonochem., 15, 350 (2008).

15. T. Ghosh, K.-Y. Cho, K. Ullah, V. Nikam, C.-Y. Park, Z.-D. Meng and W.-C. Oh, J. Ind. Eng. Chem., 19, 797 (2013).

16. Z.-D. Meng, L. Zhu, T. Ghosh, C.-Y. Park, K. Ullah, V. Nikam and W.C. Oh, Bull. Korean Chem. Soc., 33, 3761 (2012).

17. K.J. Zhang and X.H. Liu, Appl. Surf. Sci., 257, 10379 (2011).

18. X.Y. Zhang, H.P. Li, X.L. Cui and Y.H. Lin, J. Mater. Chem., 20, 2801 (2010).

19. Y. Li, X. Li, J. Li and J. Yin, Water Res., 40, 1119 (2006).

20. Z.-D. Meng, L. Zhu, K. Ullah, S. Ye, C.-Y. Park and W.-C. Oh, Asian J. Chem., 25, 5727 (2013).

21. T. Ghosh, J.-H. Lee, Z.-D. Meng, K. Ullah, C.-Y. Park, V. Nikam and W.-C. Oh, Mater. Res. Bull., 48, 1268 (2013). 